\title{
BACK TO BASICS: SOCIALLY FACILITATED SITUATED COGNITION
}

\author{
Ricardo Fonseca and Teresa Garcia-Marques \\ Instituto Superior de Psicologia Aplicada - University Institute, Lisboa
}

\begin{abstract}
A widely ignored finding in social facilitation suggests that the mere presence of others increases the "spreading out" of one's thoughts (Allport, 1920). Here, we revisit this finding and expand upon it using a situated cognition perspective.

Experiment 1 approached the spreading-out-of-thought effect using the same free-association task as Allport. Results replicated and extended previous findings. Compared to an alone condition, co-action and mere presence activated more associations, being that these associations are more context-related and more distant in the target word associative network.

Assuming that this spreading-out-of-thought effect arises from an increased salience of context-related processing, we tested this hypothesis using the Framed-Line Test paradigm in Experiment 2. Results showed that, as expected, co-action increased accuracy of estimation judgments that required incorporation of contextual information in processing.

These results support and extend Allport's view that presence of others broadens our thoughts. We discuss this idea, suggesting that social contexts may prompt cognition to be more situated.
\end{abstract}

Recent situated cognition approaches within social psychology (Levine, Resnick, \& Higgins, 1993; Semin \& Cacioppo, 2009; Smith \& Semin, 2007) have integrated the ideas developed in other fields (e.g., Arkin, 1998; Barsalou, 2003; Bredo, 1994; Glenberg, 1997; Greeno, 1989; Lave, 1988; Tulving, 1983) and suggested that cognition cannot be separated from its context. One highly relevant context is the "social context." Although it can be approached by different fields, the impact of the social context in thinking has its more direct relevance in the social facilitation phenomenon. In 1920, Allport demonstrated that we think differently when we are alone versus in the presence of others. Specifically, he observed, using a freeassociation task, that the mere presence of a co-actor increases the tendency to re-

\footnotetext{
This research was supported by a Fundação para a Ciência e Tecnologia Grant SFRH/ BD/30515/2006.

The authors thank Leonel Garcia-Marques, Diane Mackie and Debora Ko for their comments in earlier versions of this article.

Correspondence concerning this article should be addressed to Ricardo Fonseca, ISPA - IU, Rua Jardim do Tabaco, 34,1100 Lisboa, Portugal. E-mail: rfonseca@ispa.pt.
} 
spond based on the context, rather than on the stimulus itself. That is, in co-action, individuals increased the number of associations between the stimulus and its context, furnishing, for example, the associate "classroom" (instead of "educated") to the stimulus probe "teacher."

Allport suggested that this occurs because in the presence of others, there is "some sort of attitude... which takes him [the individual] 'out of himself' and directs his ideas toward outside objects" (p. 167). In our view, this interpretation clearly reflects the current idea that cognition is modulated by the degree with which it integrates the context (e.g., Blair, 2002; Higgins \& Stangor, 1988; Levine, Resnick \& Higgins, 1993; Schwarz \& Bless, 1992; Semin \& Cacioppo, 2009; Smith \& Semin, 2007). In this sense, the "sort of attitude" suggested by Allport could be best understood as a tendency to incorporate contextual information in cognitive processing when others are present.

In this paper, we will focus on and test the idea that the presence of others triggers the broadening of thought in order to incorporate contextual information in cognitive processing.

\section{SOCIAL FACILITATION AND CONTEXT-SENSITIVITY}

Research has thoroughly demonstrated that mere presence contexts, such as the mere physical presence of others or non-competing co-action, can either improve or impair performance on tasks, an effect known as social facilitation (e.g., Guerin, 1993; Zajonc, 1965). A myriad of studies show that performance on tasks like turning fishing reels (Triplett, 1898), driving (e.g., Yinon \& Levian, 1995), or impression formation (Thomas, Skitka, Christen \& Jurgena, 2002) can be affected by the mere presence of others.

One of the first empirical studies of this phenomenon (Allport, 1920, Experiment two) suggested that this passive presence of others (PPO) influences access to information available in memory. When asking participants to write as many associations to a cue as possible, Allport verified that participants produced more associations in the presence of a co-actor (i.e., another individual doing the same task). Overall, as Allport attested, these associations were more "suggested by the immediate surroundings" (i.e., more context-related). Conversely, individuals in isolation produced fewer associations, and these associations were more "suggested by the stimulus word." Allport's original interpretation of this data was that the presence of others both energizes performance and increases the tendency for individuals to be taken "out of themselves." Perhaps because of the appealing nature of this "energizing" interpretation, Allport's additional reference to context sensitivity has largely been ignored. In fact, research in social facilitation has mainly focused on the contrast between "energizing" (motivational) and "capacity" (cognitive) explanations (e.g., Baron, 1986; Zajonc, 1965, but see Lambert et al., 2003) and has disregarded this specific feature of the data that calls our attention to how the presence of others changes the relevance of the context. Here, we want to revisit this social presence-driven context sensitivity within a situated cognition approach.

In Allport's view, sensitivity to context means that: a) an environment of active persons is more likely to interfere with one's train of thought than an environment of mere space and furniture; and that $b$ ) the presence of others increases the tendency to direct one's thoughts toward stimuli outside of the self, including the 
actual presence of others. In this sense, presence of others is expected to situate cognition in our actual context, having a functional purpose of making us more prone to what is socially relevant in a situation outside of ourselves.

Thus, it may be possible that social facilitation is a phenomenon that clearly translates the idea that human cognition is modulated by the social context, being an emergent property of the person-situation interaction (e.g., Semin \& Cacioppo, 2008; Smith \& Semin, 2007). By assuming that the presence of others increases context sensitivity, we are assuming that memory, perception, and judgment are affected by situational characteristics (for reviews, see Barsalou, 2008; Greeno, 1994). A context sensitivity hypothesis can also be found in social facilitation research that shows that accompanied individuals have better recall for contextual aspects of the task situation (Sanders, Baron, \& Moore, 1978) and make greater use of contextual cues when forming an impression of a target (Thomas et al., 2002).

\section{A FIRST APPROACH TO SOCIAL FACILITATION AS AN EFFECT OF CONTEXT SENSITIVITY}

In order to offer a first approach to explore Allport's interpretation of social facilitation as related to context sensitivity and to do it within a situated cognition perspective, we designed two studies. These two studies offer a bridge between Allport's work and more recent perspectives on how context is integrated into cognition.

Our first experiment replicates and improves Allport's (1920) original study, adding information about associative commonality and overcoming some of its methodological flaws (see Guerrin, 1993). This associative commonality refers to the frequency with which a given associate of a word cue is activated in a population in general and is retrieved by norms previously defined. Adding the associative-commonality measure is particularly relevant to infer spreading of thought because it maps the associate distance in a socially shared associative network (Collins \& Loftus, 1975). The greater the distance, the lower the commonality. Thus, this measure can help us clarify Allport's results, suggesting that the presence of others changes individuals' thinking by broadening their focus beyond stimuli-specific features.

Our second experiment directly tested the idea that by spreading our thoughts, the presence of others increases individuals' attention to external context features, increasing context sensitivity. In this experiment, we aim to show that external context cues are likely to be incorporated into individuals' judgments when they are in the presence of others.

\section{EXPERIMENT 1}

Here, we replicate Allport's (1920) second experiment, by adding information about the mental representation that supports individuals' performance. Namely, we added information about the network of associates that support individuals' free association on a task.

In this way, we integrated Allport's spreading-out-of-thought hypothesis with the spread-of-activation models (e.g., Anderson, 1983; Collins \& Loftus, 1975), 
making specific assumptions about how the activation of the associative network is influenced by the presence of others. We expected the co-action group free association task outputs to have: a) a higher number of associations, but b) be less stimulus-related and more context-related (based on Allport's measure of "typeof-associate"). Also, we expect (in addition to Allport's work) these associates to be generally less common (more distantly represented in the network). By assuming that a co-action condition activates less common associations, we assume that these individuals will access more distant information represented in the network.

In modifying Allport's original study, we included a mere presence condition where we expected to replicate the effects observed with co-action. This generalization is sustained by all the literature that followed Allport's work, suggesting that mere presence is the necessary condition for the presence effect to be observed (Zajonc, 1980). We expect no differences between the two presence conditions on the effects described above.

\section{METHOD}

Participants and Design. Eighty-six ISPA (Instituto Superior de Psicologia Aplicada) undergraduate students (60 female; Mean age $=23.83 ; \mathrm{SD}=8.34$ ) received credit for their participation. Participants were randomly assigned to three levels of the social context manipulation (co-action vs. mere presence vs. isolation).

Material and Procedure. Upon their arrival at the laboratory, participants were greeted and seated in front of an IBM computer. Participants were informed that the experiment concerned how people deal with paper/pencil and computer tasks simultaneously; they would therefore receive their instructions via computer, but their responses were to be done in paper/pencil format. Moreover, participants were told to use headphones that would signal with a beep when they were to begin and stop the task. The experimenter then instructed them to start the experiment and left the room, leaving participants in one of three conditions defined by Guerin's (1993) operationalization. In the co-action condition, participants performed the task in the presence of other participants. In the mere presence condition, participants performed the task in the presence of a confederate who was reading a magazine. Finally, in the isolation condition, participants performed the task alone, without any human presence. In both presence conditions, participants were informed that their performance was anonymous and that the goal of the experiment was not to compare performance between participants, but rather to understand how people perform computer and paper/pencil tasks at the same time. This was done in order to rule out potential competition effects. For the participants in the isolation condition, these instructions were not given.

On the computer, participants were presented with general instructions on how to perform the task. Modeled after Allport's investigation (1920, Experiment two), participants were asked to put on the headphones and write down, for three minutes, all the words that came to mind when they read the word "Intelligent" (a word-cue for which associative commonality had been normalized; see Valchev, Garcia-Marques, \& Ferreira, 2005).

After five minutes (performance duration pre-tested), the experimenter returned to the room, debriefed, and thanked participants. 


\section{RESULTS AND DISCUSSION}

Number of Associates. Planned analyses tested the hypothesis that co-action and mere presence conditions would generate a greater number of associations compared to the isolation condition $(+1 ;+1 ;-2)$. Results replicate Allport's findings, suggesting that co-action $(M=14.52 ; S D=4.95)$ and mere presence $(M=16.85 ; S D$ $=6.51)$ produced more associates than isolation $(M=11.98 ; S D=5.33), t(83)=2.82$; $p=.006, d=.62$. Additional analysis showed no significant differences between the presence conditions $(+1$ co-action; -1 mere presence; isolation $=0), t(83)=1.61$; $p=.11, d=.67 ;(1-\beta)=.99 .{ }^{1}$ Type of Associate. Based on Allport's (1920) typology, five independent judges categorize each associate $(n=654)$ as a: 1$)$ cue-related associate (e.g., "bright"); 2) context-related associate (e.g., "classroom"); or 3) nonrelated associate (e.g., "beans"). The inter-judgment coefficient of agreement was considerably high $(\mathrm{K}=.72)$, and distribution of responses for these categories was further analyzed.

To test the tendency for associations to spread throughout the cognitive system in the passive presence of others (PPO), we calculated the proportion of each type of associate for each condition and contrasted them directly on our hypotheses. ${ }^{2}$ According to our rationale, we expected less cue-related associates (e.g., "smart") and more context-related associates (e.g., "classroom") in the PPO conditions than in isolation (Factor 1: mere presence $=+1$; co-action $=+1$; isolation $=-2$; Factor 2: cuerelated $=-1$; context-related $=+1 ;$ non-related $=0$ ). The results suggest that this interaction is significant, $t(83)=3.187 ; p=.003, d=.70$. Thus, these data seem to replicate the pattern obtained by Allport and generalize it to a mere presence condition (see Figure 1). Additionally, no differences emerged regarding the non-related associates between PPO and isolation $($ Mco-action $=.26 ; S D=.04 ;$ Mmere presence $=.34$; $S D=.04$ vs. Misolation $=.26 ; S D=.05, t<1)$, and no differences emerged between the two presence conditions on the three types of associates $(t ' s<1)$.

Because PPO conditions activated more associates in general, one could argue that the greater access to more context-related associates is a consequence of a more expanded network and that the use of relative frequencies is not a proper way to control for differences in the number of associates generated in each condition. To address this hypothesis, we ran the same analysis with absolute frequencies ${ }^{3}$ as dependent measures and the number of associates as covariate (highly related with our dependent measure $F(1,82)>2000 ; p=.000)$. Results demonstrated that the focused contrast is still significant, $t(82)=1.79 ; p=.03$ (one-tailed), $d=.40$ and that the conditions did not differ in the number of non-related associates $(t(82)<1)$. Together, these results suggest that the effect of the covariate accounts for some of the variance explained by the PPO, but it does not explain the effect by itself.

\footnotetext{
1. Power was computed for the contrasts that revealed to be non-significant and $t>1$.

2. General analysis suggests a main effect of type of associate, $F(1,166)=4.64 ; p=.01$. Participants produced more cue-related associates $(M=.41, S D=.03)$, followed by context-related $(M=.31, S D=$ $.03)$ and non-related $(M=.29, S D=.02)$. Moreover, the presence $x$ type-of-associate interaction was also significant, $F(4,166)=3.51 ; p=.008)$.

3. The analysis that uses absolute frequencies with no covariate reveals the same effects observed with relative frequencies, being the first contrast $t(83)=2.09 ; p=.04$, and the second one $t(83)=1.79 ; p$ $=.08$. This last marginal effect is associated with the fact that co-action increasing of responses is more clearly noticed in this type of responses (non-related associates). Results turned clear when number of associates is introduced as a co-variate.
} 


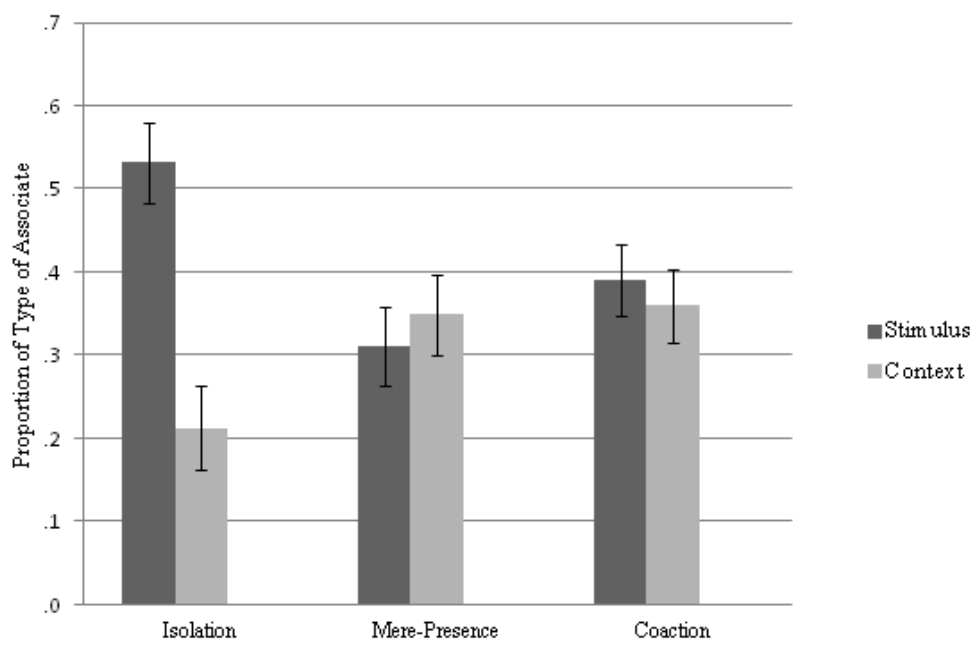

FIGURE 1. Performance in terms of types of associates as a function of the presence conditions. Error bars represent standard errors of the mean.

Associative Commonality. According to Deese (1962), one possible way to classify associations in terms of the relations among the responses to a stimulus is to "weigh the common associations by their frequency" (p. 164). To do so, we assigned each associate one of the four intervals of frequency ${ }^{4}$ of the Portuguese population norms (Valchev et al., 2005) for our target word. Then, we calculated the proportion of associates in each interval and multiplied it by its corresponding weight. Weights were as follows: 4 (for more common associates, i.e., associates with commonality above $5 \%$ ); 3 (for associates with commonality between $5 \%$ and $1 \%$ ); 2 (for associates with commonality between $0.5 \%$ and $1 \%$ ), and 1 (for less common associates, i.e., below $0.5 \%$ ). Finally, we averaged these proportions and computed an index of commonality. According to our hypothesis, if the presence conditions promote a greater spread of activation, then these individuals will exhibit lower values on this commonality index.

Planned contrast analyses $($ co-action $=-1$; mere presence $=-1$; isolation $=+2$ ) revealed a significant effect $t(83)=2.47 ; p=.02, d=.54$. As predicted, the presence conditions had a significantly lower commonality index than the isolation condition $($ Mco-action $=.33 ; S D=.09 ;$ Mmere presence $=.29 ; S D=.10$ vs. Misolation $=.38$; $S D=.11)$. No significant differences emerged between the two presence conditions, $t(83)=1.47 ; p=.16, d=.32 ;(1-\beta)=.74$.

To show that this was not a mere artifact of the increased number of associates generated in PPO, we ran the same analysis with the number of responses as a covariate. As expected, the covariate is highly associated with our dependent measure, $F(1,82)=4.68 ; p=.03, d=.48$. The relevant planned contrast performed to test our assumptions lost some strength when we controlled for the number of associates generated by each participant, but more importantly, it remained signifi-

4. In this way, we rank commonalities controlling for differences in variability within each interval between different conditions. 
cant, $t(83)=1.77 ; p=.05$ (one-tailed),$d=.39$. So, these results seem to suggest that although the number of associates in PPO is related to the access of less common associations, it does not guarantee this by itself. Thus, we interpreted these results to be consistent with Allport's findings, suggesting that PPO increases "spreadingout-of-thoughts."

One possible caveat to this interpretation may be that the instructions given to participants in PPO conditions could have boosted creative thought. By informing participants in the co-action condition that our aim was "not to compare performance," we could have promoted less normative and more idiosyncratic responses in that condition. If that was the case, this could explain the main effect of presence of others in the number of associates. However, we see no reason to assume that our instructions could also explain its moderations by "type of associates." If our instructions primed PPO participants with creativity and assuming that non-related are more idiosyncratic hence more creative, shouldn't we expect the number of non-related associates to be higher in these conditions than in isolation? Evidence that the number of these associates is equal in presence and isolation conditions seems to disprove this hypothesis.

That said we assume that these data not only replicate Allport's findings, but also clearly suggest the plausibility of the idea that presence of others prompts our cognitive system to work in a more expanded network. In other words, the passive presence of another person will make cognitive processing more permeable to the integration of information less related to the focal stimulus. Thus, we hypothesize that in the presence of others, our mind is cued to perceive an item within its context. If this is true, we could also expect that in the presence of others, general context features will be more available to individuals, making their responses more sensitive to them. The next experiment aims to test this hypothesis focusing on external context features.

\section{EXPERIMENT 2}

Research has shown that individuals differ in how sensitive they are to context cues in their information processing. When processing information, field-independent individuals have been shown to rely on an internal frame of reference, while field-dependent individuals rely on an external frame of reference (Goodenough, 1976), pay greater attention to contextual information (Witkin, Goodenough \& Oltman, 1979), are more receptive to social cues (Thomas \& Griffin, 1983), and are more sensitive to relationships between objects and the field (Nisbett, Peng, Choi, \& Norenzayan, 2001).

Among the paradigms utilized to show this effect, Kitayama, Duffy, Kawamura, and Larsen (2003) developed a paradigm that offers a helpful test for the assumption we wanted to test in our investigation. The Framed-Line Test paradigm was developed to test the assumption that North Americans are more field independent compared to their Asian counterparts. In this task, individuals were presented with a square frame within which a vertical line extended downward from the center of the upper edge of the square. Then, individuals were presented with an empty square frame and asked to draw a line identical to the first line in either absolute length (absolute task) or proportional to the height of the surrounding frame (relative task). In the absolute task, individuals had to ignore both 
the first frame (when assessing the length of the line) and the second frame (when reproducing the line). In the relative task, the participants needed to incorporate the height information of the surrounding frame in both encoding and reproducing the line. Kitayama and colleagues' results showed that North Americans, who were expected to be more field independent, performed better on the absolute task (that required ignoring both the first and the second frame) than Asians. Conversely, Asians, who were expected to be more field dependent, performed better on the relative task (that required the incorporation of the height information).

Note that the frame of the line in this task is the context, relevant to good performance on the relative task and irrelevant to the performance on the absolute task. Taking into account the results we obtained in Experiment 1 (and that no differences were found regarding non-related associates), we should expect a context relevant to the task to be more susceptible to the influence of PPO.

Thus, in our second experiment, the use of the Framed-Line Test allows us to directly test our assumption that the presence of others increases "field dependence" when the field is task-related. We expect that individuals in the PPO will perform the relative task more accurately when they have to incorporate the height information of the surrounding frame in both encoding and reproducing the line.

\section{METHOD}

Design and Participants. Thirty-one ISPA undergraduate students (20 female; Mean age $=22.51 ; S D=7.34$ ) received credit for their participation. Participants were randomly assigned to a 2 (co-action vs. isolation) $\times 2$ (absolute vs. relative task) $\times 5$ (stimulus version: the five combinations of frames and line) mixed design, in which task and version were within-subjects variables and presence was a between-subjects factor.

Material and Procedure. A replication of the Framed-Line Test paradigm developed by Kitayama and colleagues (2003) was used to test our hypothesis. Each participant was asked to draw a line in either an absolute or relative version of the task. To avoid the potential priming effects of the creative thought of Experiment 1 , both groups received the same instructions, and no reference to performance comparison or anonymity was made.

In the absolute task, participants were instructed to draw a line that was the same absolute length as the line in the first frame. In the relative task, participants were instructed to draw a line whose proportion to the size of the second frame was the same as the proportion of the first line to the size of the first frame. Before the experiment began, concrete examples were given to make sure participants understood how to perform the task. The type of task was counterbalanced for all participants.

The same five combinations used by Kitayama and colleagues (2003) of the relative sizes of the two frames and the line in the first frame were constructed. Similar to the original experiment, we created two combinations in which the first frame was larger than the second, and two other combinations in which the first frame was smaller than the second. In half of these cases, the first line was longer than one half the height of the first square. In the remaining half, the first line was shorter than half the height of the first square. In the fifth combination, the first and the second frames had the same size. 
To avoid iconic memory effects, we presented the first frame for 5 seconds on the computer screen and the second frame in paper format, where the correspondent second frame was printed. Participants received specific instructions for each task ("draw a proportional line" vs. "draw an equal line") right before they performed it. The five combinations were presented in a random order and the same stimuli were used for both the relative and the absolute tasks.

Like in Experiment 1, participants were randomly assigned to a condition in which they performed the task either in the presence of co-actors or alone in the room. In either case, the experimenter was not in the room.

\section{RESULTS AND DISCUSSION}

Performance accuracy in both tasks was assessed by measuring the lines drawn by the participants and calculating the absolute and relative differences between these lengths and the correct lengths. As in Experiment 1, since our hypothesis was directly translated in the effects associated with the general ANOVA model, we used it to test them. The mean error scores were then entered in an ANOVA with the presence vs. isolation conditions as between-subjects variables and type of task (absolute vs. relative) as within-subject factors. ${ }^{5}$

Results revealed the expected significant interaction between the type of task and the presence condition, $F(1,29)=7.97 ; p=.009, d=1.05$ (see Figure 2 ). Whereas for the relative task, participants in co-action outperformed participants in the isolation condition (Mco-action $=-.08, S D=.18$ vs. Malone $=.59, S D=.19 ; t(29)=2.62$; $p=.001 ; d=.97)$, as we expected, a different pattern characterized performance in the absolute task. When the context was not relevant to the absolute task, PPO participants seemed to be less influenced by it. That is, the data suggests that there was no reliable difference between individuals in isolation and in co-action regarding performance on the absolute task (Malone $=.49, S D=.27$ vs. Mco-action $=$ $.85, S D=.24, t(29)=1.02 ; p=.32 ; d=.38 ;(1-\beta)=.30) .{ }^{6}$ Further results also revealed a main effect of type of task, $F(1,29)=4.98 ; p=.03, d=.82$, suggesting that, overall, participants performed the relative task better $(M=.26, S D=.14)$ than the absolute task $(M=.67, S D=.18)$.

As expected, these results suggest that accompanied individuals were more accurate when they had to incorporate contextual information on judgments about a focal stimulus. However, when the contextual information is not relevant, both conditions are influenced equally by it.

This evidence corroborates our idea that the presence of others increases relevant context sensitivity. When others are present, our cognitive processes are more influenced by the situation or context, even when the stimuli are abstract rather than social.

5. The same analysis having the stimulus version (the five combinations of frames and line) as within-subject variables showed that the focused effects did not depend on the stimulus version $(\mathrm{F}<1)$, which means that the error spread equally across the five combinations (even when the two frames had the same size). Similarly, the results were not affected by the order of the task $(\mathrm{F}<1)$.

6. The reduced level of power of this analysis may suggest that although not relevant to the task context is less prone to influence individuals judgments in PPO, context sensitivity may open it to some levels of bias. 


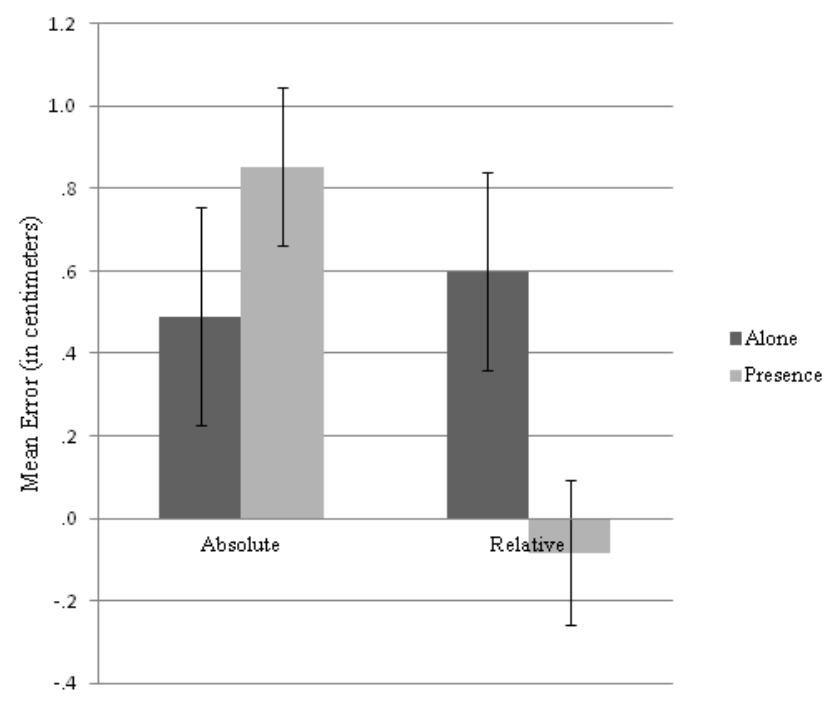

FIGURE 2. Performance on both versions of the Frame Line Test as a function of the presence condition. Error bars represent standard errors of the mean.

\section{GENERAL DISCUSSION}

Our experiments replicate and extend Allport's idea that in the presence of others, we tend to spread our thoughts, making indirect and contextual information more available in our minds. Besides replicating and extending Allport's data, we clearly showed that the presence of others increases how individuals integrate internal context features (activate more distant nodes of their mental representation) and external context features (frames of the lines to be judged) into their thoughts. In this way, this investigation supports our claim that in the PPO, individuals broaden their thinking and integrate more context features into their thoughts.

Our context-sensitivity hypothesis is not anchored in an assumption that individuals in the presence of others are necessarily more biased by undesirable and irrelevant context cues. On the contrary, data from Experiment 1 suggest that participants in the presence of others and in the isolation condition did not differ in their responses to "non-related associates." In Experiment 2, the tendency is the same; participants in the presence of others do not seem to be more biased by irrelevant contextual information than participants alone. It is worthwhile, however, to note that if an increase in statistical power detected some differences between conditions in the absolute task, that would suggest that the increased context sensitivity associated with the PPO would also suggest less accuracy, hence, more bias. What our findings suggest is that participants seem to differ in the degree to which they attend to less focal yet task-relevant context features (although possible, it is less likely that an increased sensitivity to context in PPO also biases judgment).

We have interpreted the results of Experiment 1, suggesting that PPO's decreased commonality is related to an increased sensitivity to the context of the concept that was primed. However, the decreased commonality may also be understood as increasing idiosyncratic responses. Although this could call into question our 
idea that PPO makes our cognition more situated, we believe that this is not the case. First, because decreased commonality derives from the activation of both less directly related associates and non-related associates being only the latter truly idiosyncratic (because they are scarcely socially shared). Second, because no effects emerged regarding these non-related associates across conditions in Experiment 1. Differences were observed in the increase of less direct associates.

It should be stressed that Matlin and Zajonc (1968) report what apparently seem to be contradictory results, given that they report an increase of more common responses in PPO. However, the methodological differences between our experiments and theirs seem to be highly relevant. First, Matlin and Zajonc's task requires only one first association - that is, participants are only asked to give one associate for each presented word, not allowing them to navigate in their internal representation network, which was the aim to test our hypothesis. Second, their experimental conditions were not purely social or alone, employing a withinfactor design and finding effects only in the alone condition. Third, their commonality score, instead of being reported as norms, was reported as the frequency the word had in the sample of responses generated. Their uniqueness measure was calculated based on whether the word was or was not present in PalermoJenkins' norms. It is not possible to infer context sensitivity from these data given their methodology. Corroborating our analysis, Blank (Blank, 1980; Blank, Staff, \& Shaver, 1976) showed that even though participants in the PPO give more common responses, this happens because they tend to suppress unique/idiosyncratic responses and not because they have stronger unique links to more strongly associated information. Thus, although we recognize the interest in exploring and contrast these experiments with our Experiment 1, studies such as these do not inform our context sensitivity hypothesis.

A more relevant question to our approach looks at the way we define "context." In Experiment 1, we refer to "context" as the nodes that are more distant but are incorporated in the same associative network. In Experiment 2, we refer to the line frame that needs to be attended to in order to perform the task as the context of the focal stimulus. It could be argued that the network is the definition of the concept itself and that the frame is part of the problem and so it is as important as the line to be evaluated. If that is the case, we can assume that instead of increasing context sensitivity, what PPO does is to focus individuals on the task itself. This interpretation is congruent with the one used to explain why PPO reduces Stroop interference. The explanation has been that PPO increases stimulus-relevant processing at the expense of peripheral (i.e., contextual-related) processing (Dumas, Huguet, Monteil, \& Ayme, 2005; Huguet, Galvaing, Monteil, \& Dumas, 1999). This explanation seems intriguing in light of our results, since this explanation cannot explain our findings for both studies. This offers an interesting topic to pursue in future research. Why don't PPO's participants reduce the number of non-related associates relative to those in the isolation condition in Experiment 1, and why do they not perform better than those in isolation in the absolute tasks in Experiment 2?

One possible reason why Stroop experiments offer apparently contradicting results from the ones reported in this paper may be that accompanied individuals (cueing context sensitivity) are simultaneously also more focused on the focal stimulus and aware of contextual aspects of the situation. This idea may be corroborated by some of Klauer, Herfordt, \& Voss's (2008) findings associated with the PPO's impact on Stroop effects. Klauer and colleagues suggested that the re- 
sults of both Huguet and colleagues and Dumas colleagues could be explained because a dual-task situation was used (i.e., in those experiments, participants had to form an impression of the Stroop task while performing it). According to Klauer and colleagues, PPO can influence performance at a task-selection level, rather than at the stimulus level. In support of this, Klauer and colleagues found the Stroop reduction only when individuals performed two tasks simultaneously, but not one at a time. These results may suggest that the presence of others influences how much individuals attend to the duality imposed by the context. Thus, the possibility that others may act as a conjoint cue together with the stimulus for the activation of the context features is an empirical question that future research should help to clarify.

Social facilitation effects have been classically explained by motivational factors (e.g., Baumeister, 1982; Blascovich, Mendes, Hunter, \& Salomon, 1999; Zajonc, 1965). Hence, one has to consider the possibility that our effects can be explained by the fact that those in the isolation condition are less motivated to perform the task. Consequently, they would be less willing to "explore" the situation and thus extend their attention to the context. If motivational differences could explain why PPO increases the production of associates, these differences are not able to account for the other results (e.g., the differences in the type of associates). Additional assumptions should be made to explain why motivational differences would change accuracy in contextual-embedded judgments (Experiment 2).

We believe that our hypothesis suggests a different and nuanced way of interpreting how PPO impacts judgments and behaviors: it increases context sensitivity. The question that follows these results is why PPO increases context sensitivity. One possible explanation may lie in the assumption that others may act as primes, cueing our memory system to activate more socially related information (and thus more diverse information). A caveat of this hypothesis is that it assumes spread-of-thought to be restricted to a specific context-the one related to social cues (Thomas et al., 2002). However, our experiments suggest that PPO effects are not restricted to the integration of contextual features that are social per se. Another possibility is the assumption that PPO directs individuals' attention to information that is not self-related. Congruent with this view, some approaches to social facilitation (e.g., Sanders, 1981) have shown that PPO increases attention to situational features. However, PPO has also been shown to increase self-attention (Bond, 1982), and our studies have furnished evidence of increased sensitivity only relevant to the task context. A more pure situational perspective would assume that the task to be performed is perceived differently in PPO than in isolation because our cognitive activity integrates the environmental situation that we are in. By doing that, several differences occur that are not explained by specific cognitive or specific motivational processes but rather by adaptive processes. The cognitive system adapts to whatever context it is in. As such, the presence of others, like variables such as culture, seems to promote differences in the way we deal with the situation.

We believe that the effects presented here indirectly furnish the first functional approach to the social facilitation effect, which assumes that our cognitive system is attuned to the social context in order to adapt individuals to their environment.

We are aware that many more questions can be raised and criticisms made to our set of experiments, especially given the extension of data regarding social facilitation effects. There is extensive work to be done in order to explore our claim. The 
goal of this paper is to show that there is evidence worthy of further exploration into an innovative idea that reconciles the oldest topic in social psychology with new conceptions of human behavior and thinking - the idea that even basic social contexts, such as the mere presence of others, are sufficient in making our cognition more situated.

\section{REFERENCES}

Allport, F. H. (1920). The influence of the group upon association and thought. Journal of Experimental Psychology, 3, 159-182. doi:10.1037/h0067891

Anderson, J. H. (1983). A spreading activation theory of memory. Journal of Verbal Learning and Verbal Behavior, 22, 261-295. doi:10.1016/S0022-5371(83)90201-3

Arkin, R. C. (1998). Behavior-based robotics. Cambridge, MA: MIT Press.

Baron, R. S. (1986). Distraction-conflict theory: Progress and problems. In L. Berkowitz (Ed.), Advances in experimental social psychology (Vol. 19, pp. 1-40). New York: Academic Press. doi:10.1016/S00652601(08)60211-7

Barsalou, L. W. (2003). Abstraction in perceptual symbol systems. Philosophical Transactions of the Royal Society of London: Biological Sciences, 358, 1177-1187. doi:10.1098/rstb.2002.1319

Barsalou, L.W. (2008). Grounded cognition. Annual Review of Psychology, 59, 617-645. doi:10.1146/annurev. psych.59.103006.093639

Baumeister, R. F. (1982). A self-presentational view of social phenomena. Psychological Bulletin, 91, 3-26. doi:10.1037//00332909.91.1.3

Blair, I. V. (2002). The malleability of automatic stereotypes and prejudice. Personality and Social Psychology Review, 6, 242-261. doi:10.1207/S15327957PSPR0603_8

Blank, T. O. (1980). Observer and incentive effects on word-association responding. Personality and Social Psychology Bulletin, 6, 267-272. doi:10.1177/014616728062013

Blank, T. O., Staff, I., \& Shaver, P. (1976). Social facilitation of word associations: Further questions. Journal of Personality and Social Psychology, 4, 725-733. doi:10.1037/0022-3514.34.4.725
Blascovich, J., Mendes, W. B., Hunter, S. B., \& Salomon, K. (1999). Social facilitation as challenge and threat. Journal of Personality and Social Psychology, 77, 68-77. doi:10.1037/0022-3514.77.1.68

Bond, C. F. (1982). Social facilitation: A selfpresentational view. Journal of Personality and Social Psychology, 42, 1042-1050. doi:10.1037/0022-3514.42.6.1042

Bredo, E. (1994). Reconstructing educational psychology: Situated cognition and Deweyan pragmatism. Educational Psychologist, 29, 23-35. doi:10.1207/ s15326985ep2901_3

Collins, A. M., \& Loftus, E. F. (1975). A spreading-activation theory of semantic processing. Psychological Review, 82, 407428. doi:10.1037/0033-295X.82.6.407

Deese, J. (1962). On the structure of associative meaning. Psychological Review, 3, 161175. doi:10.1037/h0045842

Dumas, F., Huguet, P., Monteil, J. M., \& Ayme, E. (2005). Context effects in the Stroop task: When knowledge of one's relative standing makes a difference. Current Psychology Letters: Behaviour, Brain \& Cognition, 16, 1-12. Retrieved from http:/ / cpl.revues.org/index456.html

Glenberg, A. M. (1997). What memory is for. Behavioral and Brain Sciences, 20, 1-55. doi:10.1017/S0140525X97000010

Goodenough, D. R. (1976). The role of individual differences in field dependence as a factor in learning and memory. Psychological Bulletin, 83, 675-694. doi10.1037/0033-2909.83.4.675

Greeno, J. G. (1989). A perspective on thinking. American Psychologist, 44, 134-141. doi:10.1037/0003-066X.44.2.134

Greeno, J. G. (1994). Gibson's affordances. Psychological Review, 2, 336-342. doi:10.1037/0033-295X.101.2.336 
Guerin, B. (1993). Social facilitation. Cambridge: Cambridge University Press.

Higgins, E. T., \& Stangor, C. (1988). A “changeof-standard" perspective on the relations among context, judgment, and memory. Journal of Personality and Social Psycho$\log y, \quad 54$, 181-192. doi:10.1037/00223514.54.2.181

Huguet, P., Galvaing, M. P., Monteil, J. M., \& Dumas, F. (1999). Social presence effects in the Stroop task: Further evidence for an attentional view of social facilitation. Journal of Personality and Social Psycho$\log y$, 77, 1011-1025. doi:10.1037/00223514.77.5.1011

Kitayama, S., Duffy, S., Kawamura, T., \& Larsen, J. T. (2003). A cultural look at New Look: Perceiving an object and its context in two cultures. Psychological Science, 14, 201-206. doi:10.1111/14679280.02432

Klauer, K. C., Herfordt, J., \& Voss, A. (2008). Social presence effects on the Stroop task: Boundary conditions and an alternative account. Journal of Experimental Social Psychology, 44, 469-476. doi:10.1016/j. jesp.2007.02.009

Lambert, A. J., Payne, B. K., Jacoby, L. L., Shaffer, L. M., Chasteen, A. L., \& Khan, S. R. (2003). Stereotypes as dominant responses: On the "social facilitation" of the prejudice in anticipated public contexts. Journal of Personality and Social Psychology, 84, 277-295. doi:10.1037/00223514.84.2.277

Lave, J. (1988). Cognition in practice: Mind, mathematics, and culture in everyday life. Cambridge: Cambridge University Press.

Levine, J. M., Resnick, L. B., \& Higgins, E. T. (1993). Social foundations of cognition. Annual Review of Psychology, 44, 585-612. doi:10.1146/annurev.psych.44.1.585

Matlin, M. W., \& Zajonc, R. B. (1968). Social facilitation of word associations. Journal of Personality and Social Psychology, 10, 455460. doi:10.1037/h0026815

Nisbett, R. E., Peng, K., Choi, I., \& Norenzayan, A. (2001). Culture and systems of thought: Holistic versus analytic cognition. Psychological Review, 108, 291-310. doi:10.1037/0033-295X.108.2.291

Sanders, G. S. (1981). Driven by distraction: An integrative review of social facilitation theory research. Journal of Expe- rimental Social Psychology, 17, 227-251. doi:10.1016/0022-1031(81)90024-X

Sanders, G. S., Baron, R. B., \& Moore, D. L. (1978) Distraction and social comparison as mediators of social facilitation effects. Journal of Experimental Social Psychology, 14, 291-303. doi:10.1016/00221031(78)90017-3

Schwarz, N., \& Bless, H. (1992). Constructing reality and its alternatives: Assimilation and contrast effects in social judgment. In L. L. Martin \& A. Tesser (Eds.), The construction of social judgment (pp. 217245). Hillsdale, NJ: Erlbaum.

Semin, G. R., \& Cacioppo, J. T. (2008). Grounding social cognition: Synchronization, coordination, and co-regulation. In G. R. Semin \& E. R. Smith (Eds.), Embodied grounding: Social, cognitive, affective, and neuroscientific approaches (pp. 119-147). New York: Cambridge University Press.

Semin, G. R., \& Cacioppo, J. T. (2009). From embodied representation to co-regulation. In J. A. Pineda (Ed.), Mirror neuron systems: The role of mirroring processes in social cognition (pp. 107-120). New York: Humana.

Smith, E. R., \& Semin, G. R. (2007). Situated social cognition. Current directions in psychological science, 16, 132-135. doi:10.1111/j.1467-8721.2007.00490.x

Thomas, J., \& Griffin, R. (1983). The social information processing model of task design: A review of the literature. Academy of Management Review, 8, 672-682. doi: $10.2307 / 258268$

Thomas, S. L., Skitka, L. J., Christen, S., \& Jurgena, M. (2002). Social facilitation and impression formation. Basic and Applied Social Psychology, 24, 67-70. doi:10.1207/153248302753439146

Triplett, N. (1898). The dynamogenic factors in pacemaking and competition. American Journal of Psychology, 9, 507-533. doi: $10.2307 / 1412188$

Tulving, E. (1983). Elements of episodic memory. Oxford, UK: Clarendon.

Valchev, N., Garcia-Marques, L., \& Ferreira, M. (2005). O sonho (DREAM) comanda a memória: Listas de palavras associadas para estudos de falsas memórias [The DREAM controls memory: Word association lists to study false memories]. Laboratório de Psicologia, 3, 41-64. 
Witkin, H. A., Goodenough, D. R., \& Oltman, P. K. (1979). Psychological differentiation: Current status. Journal of Personality and Social Psychology, 37, 1127-1145. doi:10.1037/0022-3514.37.7.1127

Yinon, Y., \& Levian, E. (1995). Presence of other drivers as a determinant of traffic violations. Journal of Social Psychology, 135,
299-304. doi:10.1080/00224545.1995.971 3959

Zajonc, R. (1980). Compresence. In P. Paulus (Ed.), Psychology of group influence (pp. 35-60). Hillsdale, NJ: Erlbaum.

Zajonc, R. B. (1965). Social facilitation. Science, 149, 269-274. doi:10.1126/science.149.3681.269 
Copyright of Social Cognition is the property of Guilford Publications Inc. and its content may not be copied or emailed to multiple sites or posted to a listserv without the copyright holder's express written permission. However, users may print, download, or email articles for individual use. 